\title{
Video Error Resilience Scheme using Reversible Data Hiding Technique for Intra- Frame in H.264/AVC
}

\author{
Ranran Li, Rangding Wang ${ }^{1}$
}

\begin{abstract}
For the purpose of improving the quality of wireless video transmission, this paper proposed an error resilience scheme based on reversible data hiding to reconstruct lost blocks. The motion vector of each macroblock (MB) in IntraFrame is extracted first. Then as the important data of each MB, it is imperceptibly embedded into corresponding $\mathrm{MB}$ of the same frame using a reversible data hiding technique. If the embedded data for a corrupted MB is still available at the decoder side, the lost $\mathrm{MB}$ can be recovered using extracted important data. Otherwise, the lost MB can be recovered with a traditional error concealment method. Simulation results show that our method has higher PSNR than the reference methods in H.264/AVC.
\end{abstract}

Keywords: Error Resilience · Reversible · Data Hiding · Video transmission

\section{Introduction}

As the multimedia technology increasingly matures and user demand rises, wireless video communication is attracting more and more attention. Wireless channel is susceptible to noise, network congestion, shadow fading and multipath in the process of data transmission. Therefore it is not reliable enough to guarantee lossless transmission. Furthermore, videos own a large number of raw data. So under the condition of limited transmission bandwidth and storage capacity, the original video data must be compressed effectively. H.264/AVC adopts the variable length coding and prediction techniques to improve coding efficiency [1]. However, it leads video signals to dependent on the integrity of the stream. Even a single bit error may lead to loss of synchronization and errors can spread to other blocks in the same frame as well as subsequent frames. These cause the remaining bit-

\footnotetext{
Rangding Wang $(\bowtie)$

College of Information Science and Engineering, Ningbo University, Ningbo, China

wangrangding@nbu.edu.cn
} 
streams cannot be decoded properly, which would severely affect the quality of reconstructed video. Therefore, error resilience is utilized to solve this problem.

The data hiding based error resilience method introduces the data embedding technique into the video error resilience. At the encoder side, some important characteristics of the original video image are rapidly extracted as the important data. These data are embedded to video carrier by information hiding method which is originally proposed for content authentication [3], secret communication [4], and copyright protection [5]. And then the hidden information can be available for error detection [2], resynchronization and error recovery [6] at the decoder side. An effective error recovery for intra-frame based on data hiding in H.264/AVC is proposed by Chen et al. [7]. This error concealment method diverts computation burden from decoder to encoder which holds more effective resources. In their method, the motion estimation process is first carried out between two consecutive intra-frames and the motion vector (MV) of each MB is embedded into quantized DCT (QDCT) coefficients by odd-even data hiding method. At the decoder side, the MV of each lost MB can be extracted from the corresponding MB and the lost MB can be restored through the implementation of motion compensation. Researching on the human visual system (HVS) indicates that the region of interest (ROI) in images, such as foreground areas and moving objects, usually attract much attention. So the error concealment (EC) of the ROI receives special treatment in [8]. In this method, motion vectors of the ROI are embedded in background adaptively using odd-even data hiding method. At the decoder side, when an ROI is found missing, previously embedded MVs can be extracted to facilitate the EC of the ROI. Error resilience methods based on data hiding have many advantages, such as saving the channel resources compared with adding redundant slices methods [6] and gaining more available information at the encoder side compared with the error concealment methods. But QDCT coefficients are changed permanently during data embedding. To solve this problem, a reversible data hiding method based on histogram modification technique was adopted by Chung et al. [10]. The MV of each MB is embedded into QDCT coefficients and original data of the video can be lossless recovery after data is extracted. But according to histogram modification rules, the algorithm have to shift the histogram between the peak point and zero point to the right side by one level to create an empty bin besides the peak point for data hiding. This affects the video imperceptibility and increases the transmission rate to some extent since many of coefficients are moved. In [9], Lin et al. apply other reversible data hiding algorithm which bases on differential expansion (DE) to meet the goal of error resilient. Since four times of DE are required to embed pixel values into QDCT, computational complexity is higher.

To improve the quality of videos error recovery as far as possible, a video error resilience scheme using reversible data hiding technique for Intra-Frame in H.264/AVC is proposed in the paper. At the encoder, for an Intra-frame, reversible data embedding method based on the generalized difference expansion (GDE) is used to hide important data. At the decoder, if important data can be extracted 
correctly, the corrupted MB is recovered with extracted information. Otherwise, boundary matching method (MBA) can be operated to recover the corrupted MB.

The rest of the paper is organized as follows. Section 2 elaborates important data extraction of each MB. Section 3 focuses on the operations of proposed error resilience algorithm based on reversible data embedding. We verify the effectiveness of the proposed method with experimental results in Section 4.Section 5 makes a conclusion for the results and discusses the possible ways to improve the error recovery algorithm in the future.

\section{Important Data Extraction}

By performing motion estimation with search range \pm 15 and half-pixel accuracy between current Intra-Frame $I_{k}$ and previous Intra-Frame $I_{k-1}$, the motion vector (MV) of each MB is obtained [7]. Then motion vector is encoded into binary bits. The total number of binary bits is $\mathrm{L}=2 \times\left(\log _{2}[(2 \times 15+1)]+1\right)=12$.

A circular embedding scheme [10] is adopted to embed important data. That means, $\operatorname{MV}(i, j), M V(i, j+1), M V(i+1, j), M V(i+1, j+1)$ are embedded into $M B(i$, $\mathrm{j}+1), M B(i+1, j+1), M B(i, j), M B(i+1, j)$ respectively. Here $M V(i, j)$ is the motion vector of $\operatorname{MB}(i, j), i=\{0,2,4, \cdots \cdots, W / 16\}$ and $j=\{0,2,4, \cdots \cdots, H / 16\} . H$ and $W$ are defined as the height and width of a video frame.

\section{Proposed Error Resilience Algorithm Based on Reversible Data Embedding}

\subsection{Reversible Data Hiding Scheme Based on GDE}

In this paper, a reversible data hiding method based on the generalized difference expansion (GDE) is adopted to embed important information into original video carrier. GDE is evolved from DE [11] and it is based on a pair reversible integer transform.

Reversible integer transform: for the vector $X=\left(X_{0}, X_{1}, \ldots \ldots, X_{N-1}\right)$, the forward difference expansion transform $\mathrm{f}(\cdot)$ is denoted by:

$$
\mathrm{y}_{0}=\left\lfloor\frac{\sum_{i=0}^{N-1} a_{i} x_{i}}{\sum_{i=0}^{N-1} a_{i}}\right\rfloor, \mathrm{y}_{1}=x_{1}-x_{0}, \cdots, \mathrm{y}_{N-1}=x_{N-1}-x_{0}
$$


For transformed vector $\mathrm{y}=\left(\mathrm{y}_{0}, \mathrm{y}_{1}, \cdots \cdots, \mathrm{y}_{N-1}\right)$, the inverse difference expansion transform $f^{-1}(\cdot)$ is defined as:

$$
x_{0}=\mathrm{y}_{0}-\left\lfloor\frac{\sum_{i=1}^{N-1} a_{i} y_{i}}{\sum_{i=0}^{N-1} a_{i}}\right\rfloor, x_{1}=\mathrm{y}_{1}+x_{0}, \cdots \cdots, x_{N-1}=y_{N-1}+x_{0}
$$

where $\lfloor\cdot]$ is the least nearest integer and $a_{i}$ is a constant integer which is set to 1 in our method.

\subsection{Embedding Zone Selection}

There are two diverse modes according to different size of the encoding unit, i.e., Intra_4×4 and Intra_16 $\times 16$ in Intra-Frame. And then each MB is divided into sixteen $4 \times 4$ subblocks orderly (shown in Fig.3.1(a)). According to a zig-zag scanning fashion, all of the quantized coefficients with a $4 \times 4$ block can be marked orderly, from $0,1, \cdots \cdots$, up to 15 (labeled in Fig.3.1(b)).Considering DC and low frequency coefficients contain most of the energy and embedding data in them may affect the video quality and the bit-rate significantly. Therefore, for the purpose of imperceptibly embed a certain amount of information and reducing the complexity, mid- frequency and high-frequency DCT coefficients in the first $4 \times 4$ block are chosen to embed data. Selected coefficients can form a 1-dimensional vector $x=$ $\left(x_{0}, X_{1}, \cdots \cdots, X_{N-1}\right)$,which exactly includes 13 values.
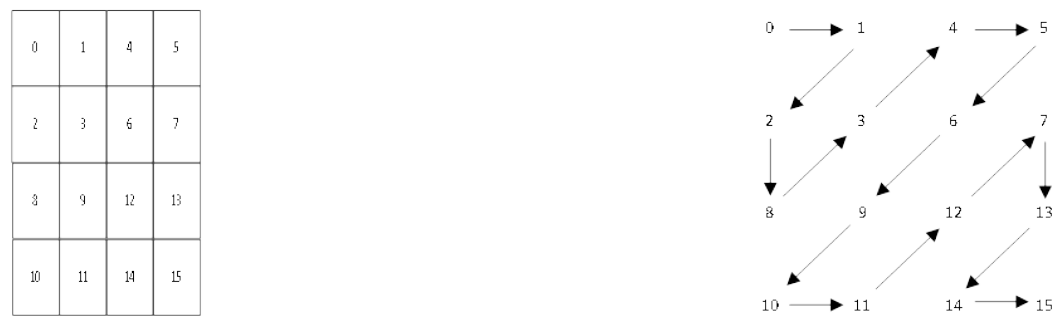

Fig.3.1(a) Scanning order of subblocks within a MB, (b)zig-zag scanning order

\subsection{Embedding Process}

The important data $w_{m v}=\left(w_{1}, w_{2}, \cdots \cdots, w_{12}\right)$ of each MB is embedded in the quantized DCT coefficients $X=\left(X_{0}, X_{1}, \cdots \cdots, x_{12}\right)$, as follows:

Step1: Calculate y using the reversible integer transform $\mathrm{f}(\cdot)$ (see Eq.(3.1)) .

Step2:Expand the vector $\mathrm{y}$ as in Eq.(3.3). Then the values of 


$$
\begin{aligned}
& \widehat{\mathbf{y}}=\left(y_{0}, y_{1}, \cdots \cdots, y_{12}\right) \text { are taken sequentially. } \\
& \quad \mathrm{y}_{0}=\left\lfloor\frac{\sum_{i=0}^{12} a_{i} x_{i}}{\sum_{i=0}^{12} a_{i}}\right\rfloor, \hat{\mathrm{y}}_{1}=2 \times \mathrm{y}_{1}+w_{1}, \cdots \cdots, \mathrm{y}_{12}=\mathrm{y}_{12}+w_{12}
\end{aligned}
$$

Step3: Perform the inverse reversible integer transform for y y using $f^{-1}(\cdot)$ (see Eq.(3.2)) to produce the watermarked $\hat{x}$.

Step4: Replace the quantized DCT values with the corresponding values in watermarked vectors $\hat{x}$.

\subsection{Extraction process}

Step1: Calculate $\hat{y}$ using the formula (3.1).

Step2: Extract the LSB of $\left|\widehat{\mathbf{y}}_{1} \uparrow,\right| \mathbf{y}_{2}|, \ldots,| \mathbf{y}_{12} \mid$ using equation (3.4).

$$
\widehat{w}_{n}=\left|\mathrm{y}_{n}\right| \bmod 2 \quad n=1,2,3, \cdots, 12
$$

Step3: The original value of each element in vector y is restored as follows:

$$
\mathrm{y}_{0}=\hat{\mathrm{y}}_{0}, \mathrm{y}_{1}=\frac{\hat{\mathrm{y}}_{1} \sim w_{1}}{2}, \cdots \cdots, \mathrm{y}_{12}=\frac{\mathrm{y}_{12}-w_{12}}{2}
$$

Step4: Perform the inverse reversible integer transform for the resulting vector using $f^{-1}(\cdot)$ to restore the original vector $x$.

Step5: Replace the values in the vector $\hat{x}_{n}$ with the corresponding values in the restored vector $x$.

\subsection{Error Resilience Process}

At the decoder side, when a MB is detected missing, it is defined as MB lost. If the embedded data for a corrupted MB is still available, the lost MB can be recovered using extracted important data. With the extracted MV of current MB and motion compensation, the best matching macroblock ( $\mathrm{MB}$ best) can be found in the previous Intra-Frame. Then $\mathrm{MB}$ lost is replaced with $\mathrm{MB}$ best .Otherwise, the lost $\mathrm{MB}$ can be recovered with boundary matching error concealment method.

\section{Experimental results and discussions}

In this paper, the H.264/AVC reference software JM-12.0 is used as simulation platform. Six standard QCIF video sequences, namely, Carphone, Container, 
Mobile, Silent, News and Foreman, are used for our simulation. 150 frames of each video sequence are encoded for the test. The period of intra-frame is 5 and the frame rate is set to $15 \mathrm{fps}$.

\subsection{Performance Evaluation of Error Recovery}

The objective evaluation criteria PSNR between original video sequences and restored video sequences against the loss of MBs are used to evaluate the performance of error recovery. In order to comparing the error recovery performance with involved methods, $0 \%, 10 \%$ and $20 \%$ random loss of Intra-frame MBs are set in this paper. The results of error recovery are shown in Table4.1. In the table, JM algorithm is the error concealment method without data hiding for intra-frame in H.264/AVC reference software JM-12.0. DHEC is Chen's error recovery algorithm [7] based on data embedding.

Table 4.1 PSNR (dB) comparison at different loss rate of intra-frame MBs

\begin{tabular}{|c|c|c|c|c|}
\hline \multicolumn{5}{|c|}{$(\mathrm{QP}=33)$} \\
\hline \multirow[b]{2}{*}{ Video } & \multirow[b]{2}{*}{ Method } & \multicolumn{3}{|c|}{ Macroblock loss rate /\% } \\
\hline & & 0 & 10 & 20 \\
\hline \multirow{3}{*}{ Carphone } & JM & 34.49 & 29.32 & 26.31 \\
\hline & DHEC & 34.38 & 32.89 & 29.83 \\
\hline & Proposed & 34.49 & 33.14 & 30.85 \\
\hline \multirow{3}{*}{ Mobile } & $\mathrm{JM}$ & 31.00 & 27.07 & 24.46 \\
\hline & DHEC & 29.89 & 27.69 & 24.68 \\
\hline & Proposed & 31.00 & 30.03 & 28.76 \\
\hline \multirow{3}{*}{ Foreman } & JM & 33.63 & 29.33 & 26.49 \\
\hline & DHEC & 33.44 & 31.22 & 28.03 \\
\hline & Proposed & 33.63 & 31.25 & 28.62 \\
\hline \multirow{3}{*}{ Container } & $\mathrm{JM}$ & 33.73 & 29.91 & 27.29 \\
\hline & DHEC & 33.41 & 32.86 & 31.97 \\
\hline & Proposed & 33.73 & 33.44 & 32.90 \\
\hline \multirow{3}{*}{ News } & JM & 33.89 & 27.87 & 25.08 \\
\hline & DHEC & 33.62 & 30.76 & 29.91 \\
\hline & Proposed & 33.89 & 31.54 & 30.07 \\
\hline \multirow{3}{*}{ Silent } & $\mathrm{JM}$ & 32.96 & 29.93 & 27.64 \\
\hline & DHEC & 32.90 & 31.66 & 30.63 \\
\hline & Proposed & 32.96 & 31.89 & 30.89 \\
\hline
\end{tabular}




\title{
4.2 Discussion
}

As described in Table 4.1, when loss rate of Intra-frame MBs is $0 \%$, that is, there are not any MBs missing, our method has no PSNR degradation. It is because that data hiding method is reversible which can restore the original video after data extraction. So it does not have any impact on visual quality of reconstructed video. The average PSNR of the proposed method is improved up to $2.97 \mathrm{~dB}$ and $0.7 \mathrm{~dB}$ compared with JM and Chen's method at loss rate $10 \%$ respectively. At loss rate $20 \%$, the average PSNR of the proposed method is also higher than the both reference method obviously.

\section{Conclusions}

Odd-even data hiding method destroys the original video data permanently, so error resilient techniques based on odd-even data hiding reduce the quality of video images in a certain degree. Therefore an error resilience using reversible data hiding for Intra-Frame is introduced in the paper.

At the encoder side, by adopting GDE technology, the proposed method embeds MV of each MB into QDCT coefficients of corresponding MB in the same frame. At the decoder side, whether the MV of missing MB can be extracted or not is firstly determined. If the embedded MV can be extracted successfully from corresponding $\mathrm{MB}$, then the hidden $\mathrm{MV}$ is extracted and used for searching its best match $M B_{\text {best. }}$ The corrupted $M B$ is recovered by being replaced with $\mathrm{MB}_{\text {best }}$. Otherwise, boundary matching method can be used to recover the lost MB.

The proposed error recovery method improves the quality of error recovery effectively, but it doesn't consider scene change circumstances in the videos. In the future, our work is to figure out the proper way to detect scene change and extract adaptively richer feature information for error recovery.

\begin{abstract}
Acknowledgement This work is supported by the National Natural Science Foundation of China (61170137), Doctoral Fund of Ministry of Education of China (20103305110002), Zhejiang Provincial Natural Science Foundation of China (Y13F020071), Key Innovation Team of Zhejiang Province (C01416124200), The Outstanding (Postgraduate) Dissertation Growth Foundation of Ningbo University (10Y20100002). Zhejiang Provincial Natural Science Foundation of China (LY13F020013). Open Fund of Zhejiang Provincial Top Key Discipline of Information and Communication Engineering (XKXL1310).
\end{abstract}

\section{References}

1. ITU-T Rec (2003). H.264 / ISO/IEC 11496-10. Advanced Video Coding. Final Committee Draft, Document JVTG050. 
2. M. G. Ko, J. E. Hong, J. W. Suh (2012). H.264/AVC Error Detection Scheme Using Fragile Data Hiding in Motion Vector Set. IEEE International Conference on Consumer Electronics (ICCE). $237-238$.

3. D.W. Xu, R.D. Wang, J.C. Wang (2011). A novel watermarking scheme for H.264/AVC video authentication. Signal Processing: Image Communication. 26(6): 267-279.

4. D.W. Xu, R.D. Wang, J.C.Wang (2012). Prediction mode modulated data-hiding algorithm for H.264/AVC. Journal of Real-Time Image Processing. 7(4):205-214.

5. A.Mansouri, A. Mahmoudi, F.Torkamani-Azar, F. Kurugollu (2010). A low complexity video watermarking in H.264 Compressed Domain. IEEE Transactions on Information Forensics and Security. 5(4):649-657.

6. J. J. Xu, W. M. Zhang,N. H. Yu, F. Zhu. B. Chen (2011). Error Resilient Coding Based on Reversible Data Hiding and Redundant Slice. Sixth International Conference on Image and Graphics (ICIG). 223-227.

7. S. Y. Chen, H. Leung (2009). A temporal approach for improving intra-frame concealment performance in H.264/AVC [J] . IEEE Transactions on Circuits and Systems for Video Technology, 19(3) :422-426.

8. Z. Y. Luo, L. Song, S. B. Zheng, Y. Xu. X. K. Yang (2010). Improved error concealment of region of interest based on the H.264/AVC standard. Optical Engineering. 49(4): 047003047003.

9. S. D. Lin, H. C. Meng, C. H. Chuang, J. Y. Huang (2008). An error resilient technique using reversible data embedding in H.264/AVC[C]. The 3rd International Conference on Innovative Computing Information and Control. 26-29.

10. K. L. Chung, Y. H. Huang, P. C. Chang, H. Y. Mark Liao (2010). Reversible data hidingbased approach for intra-frame error concealment in H.264/AVC [J]. IEEE Transactions on Circuits and Systems for Video Technology. 20(11): 1643-1647.

11. A. M. Alattar (2004). Reversible watermark using the difference expansion of a generalized integer transform. IEEE Transactions on Image Processing. 13(8):1147-1156. 